\title{
LA OCUPACIÓN INCA EN CONVENTOMOQO, VALLE DEL CUSCO
}

\author{
Carlos Delgado González \\ Departamento Académico de Aroueología, Universidad Nacional de San Antonio Abad del Cusco \\ mauriciodel@hotmail.com
}

\section{RESUMEN}

El trabajo de investigación fue parte de un proyecto de salvataje del año 2007, realizado en diversos sitios arqueológicos que vienen siendo ocupados por la creciente urbanización de las laderas del valle del Cusco.

A la llegada de los españoles a la ciudad del Cusco, ésta se encontraba densamente ocupada, tanto el núcleo central, como en los alrededores de la ciudad donde la población vivia en aldeas. Conventomoqo fue una pequeña aldea doméstica en la margen derecha del río Huatanay, que se asentó sobre una colina en un área que ya estuvo anteriormente ocupada desde el periodo Formativo. El fechado radiocarbónico obtenido en el lugar es tardio y se ubicaría en el periodo Inca-colonial, concordando con la idea que las poblaciones siguieron ocupando estos lugares hasta que el virrey Toledo, en 1572, realiza la reducción a ocho parroquias de los pueblos incas que vivían en la periferia de la ciudad. El registro arqueológico identificó varios eventos de abandono del lugar que están plasmados en el presente trabajo.

Palabras Clave: Inca, colonial, asentamiento, Cusco.

\section{ABSTRACT}

The research was part of a proposed bailout of 2007, made in one of the many archaeological sites that are being occupied by the increasing urbanization for the slopes of the valley of Cusco.

With the arrival of the Spaniards to the city of Cusco, this place was densely occupied, both the central core, as in the surrounding area of the city where the population lived in villages. Conventomoqo was a small domestic village in the the right margin of the Huatanay River, and it was settled on a hill in an area that was already previously occupied from the formative period. The radiocarbon dated obtained in the place show that this place is late and would be placed in the Inca period-colonial, agreeing with the idea that the populations continued to occupy these places until the viceroy Toledo, in 1572, he makes the reduction in eight parishes of the Inca peoples who lived on the outskirts of the city. The archaeological record identified several events of abandonment of this place that are embodied in the present work.

KEYwords: Inca, colonial, settlement, Cusco. 


\section{El valle del Cusco}

El valle del Huatanay corresponde a un área de bosque seco montano subtropical, con una estructura vegetal espinosa que es común a la mayoría de los valles interandinos.

El sistema hidrográfico y la riqueza de la napa freática del valle dieron lugar a que aparecieran los más diversos tipos de corrientes de agua, tales como ríos, arroyos, pantanos, manantes y puquiales. Es asi que el río Huatanay, que se forma por la unión de los ríos Huancaro, Chocco y Saphy, transporta el agua de manantes de la parte alta, agua de lluvia y sedimentos y los desemboca en el río Vilcanota, a la altura del poblado de Huambutio (Centro Guaman Poma de Ayala 2005: 189). Asimismo, el río Huatanay, entre Huancaro y el Aeropuerto Alejandro Velazco Astete, tiene una pendiente muy baja de solo $0,8 \%$ determinando que la zona sea inundable, desde este punto prosigue con una mayor pendiente hasta Larapa y Angostura (Centro Guaman Poma de Ayala 2005: 189). Debido a sus áreas pantanosas los Incas fueron desecando el piso del valle mediante canales de drenaje, un registro interesante de estos drenes se tiene en el sector de San Judas Grande en el distrito de Wanchaq (Ardiles 1986).

Debido a las características antes mencionadas, el piso de valle no era adecuado para una ocupación humana permanente (Bauer 2008: 21), a su vez, las zonas más productivas agrícolamente son las grandes terrazas aluviales de la parte sur que se encuentran sobre los $25 \mathrm{~m}$ del piso de valle y que son aptas para el cultivo de diferentes productos como el maíz que se produce hasta los 3600-3800 m, la papa hasta los $4300 \mathrm{~m}$ y la quinua entre los 2000-3600 $\mathrm{m}$ de altitud que se siembran generalmente con el maíz (Ugent y Ochoa 2006).

\section{LoS INCAS EN EL VAlle Del Huatanay}

Los primeros trabajos arqueológicos en el valle del Huatanay se iniciaron con Valcárcel (1934, 1935), Pardo (1939a, 1939b, 1957), Llanos (1936), Rowe (1944) y el Patronato de Arqueología del Cusco durante la década de 1970, seguidamente el Instituto Nacional de Cultura realizó trabajos de levantamientos topográficos y algunos trabajos de excavación arqueológica en diferentes sitios. El trabajo más ambicioso de reconocimiento y prospección de los asentamientos en el valle del Huatanay lo realizó Bauer en 1994 y entre los años 1998-1999, conjuntamente con Alan Covey, totalizando unos 1200 sitios identificados (Bauer 2008: 29-32). Es durante este trabajo que se identificó el sitio de Conventomoqo asociado a varios estilos cerámicos formativo y Qotakalli.

Durante el desarrollo y apogeo del estado Inca, la población ocupaba la periferia de la ciudad y las áreas con más densidad se encontraban hacia ambas margenes del río Huatanay con una mayor incidencia hacia el oeste de la ciudad en los actuales distritos de Santiago y San Sebastián.

Poco estudiadas han sido las poblaciones que ocuparon la periferia o los suburbios de la ciudad Inca. Bauer y Covey, como parte de la prospección intensiva en el valle del Cusco, identificaron más de 850 sitios incas (Bauer 2008: 190), Susan Niles prospectó hacia el norte de San Sebastián en Callachaca (1984). En las dos últimas décadas se fueron dando algunos trabajos arqueológicos en varios sitios incas como en Qasapatallacta (Candia 1992, 1995; Pilares 2002), en Silkinchani (Claros y Mormontoy 1992; Cumpa 2001), en Qotakalli (Barreda 1973; Del Pezo 2002), Wimpillay (Barreda 1973), en Lucerinas (Farfán 2009), en Muyu Orqo (Pilco 2005) y en Raqay Raqayniyoc (Niles 1984, Benavides y Vizcarra 2010) y los trabajos realizados por el Instituto Nacional de Cultura-Cusco.

Los lugares habitacionales por lo general están asociados a grupos de depósitos, terrazas de cultivo y canales de riego. El sitio de Qotakalli está conformado por 68 estructuras de forma rectangular y cuadrangular y algunas de ellas presentan divisiones interiores (Del Pezo 2002: 60; Niles 1984: 212), Silkinchani está integrado por nueve estructuras rectangulares asociadas a terrazas artificiales (Cumpa 2001: 46), el sitio de Raqay Raqayniyoc a 38 edificios, Callachaca a 13 edificios construidos a lo largo de las terrazas y Qasapatallaqta a más de 80 edificios (Niles 1984: 211-213). 
Hacia la margen izquierda del río Huatanay los sitios incas están asociados a una infraestructura agrícola como el sistema de andenes de Larapa, Patapata, Pukapukara, Patapatayoc, Kallampa, Puska, Chimparaqay. A la margen derecha tenemos Machutauqaray, Waynatauqaray, Choqo, Lloqepugio, Qontaymoqo, Mesapata, Silkinchani entre otros (Fig. 1). De esta manera para el mantenimiento y aprovicionamiento de excedentes de producción se tuvo un sistema de depósitos a lo largo de todo el valle y uno de los más grandes es el de Qasapatallacta con más de cien unidades de almacenaje (Bauer 2008: 197). Otros depósitos tenemos en Salonniyoq (Guillén 2009: 56), Muyu Orqo (Pilco 2005: 15-16, 48), en Arahuay (Quispe 2009: 123-126) y los que se encontraban en Callachaca al norte de San Sebastián.

La ciudad del Cusco, como núcleo organizador del estado inca, estuvo conformado por un conjunto de aldeas a su alrededor, uno de estos fue Conventomoqo, un área relativamente pequeña en comparación a otras aldeas grandes como Qasapatallaqta, Wimpillay, Qotakalli y Raqay-Raqayniyoc (Fig. 1).

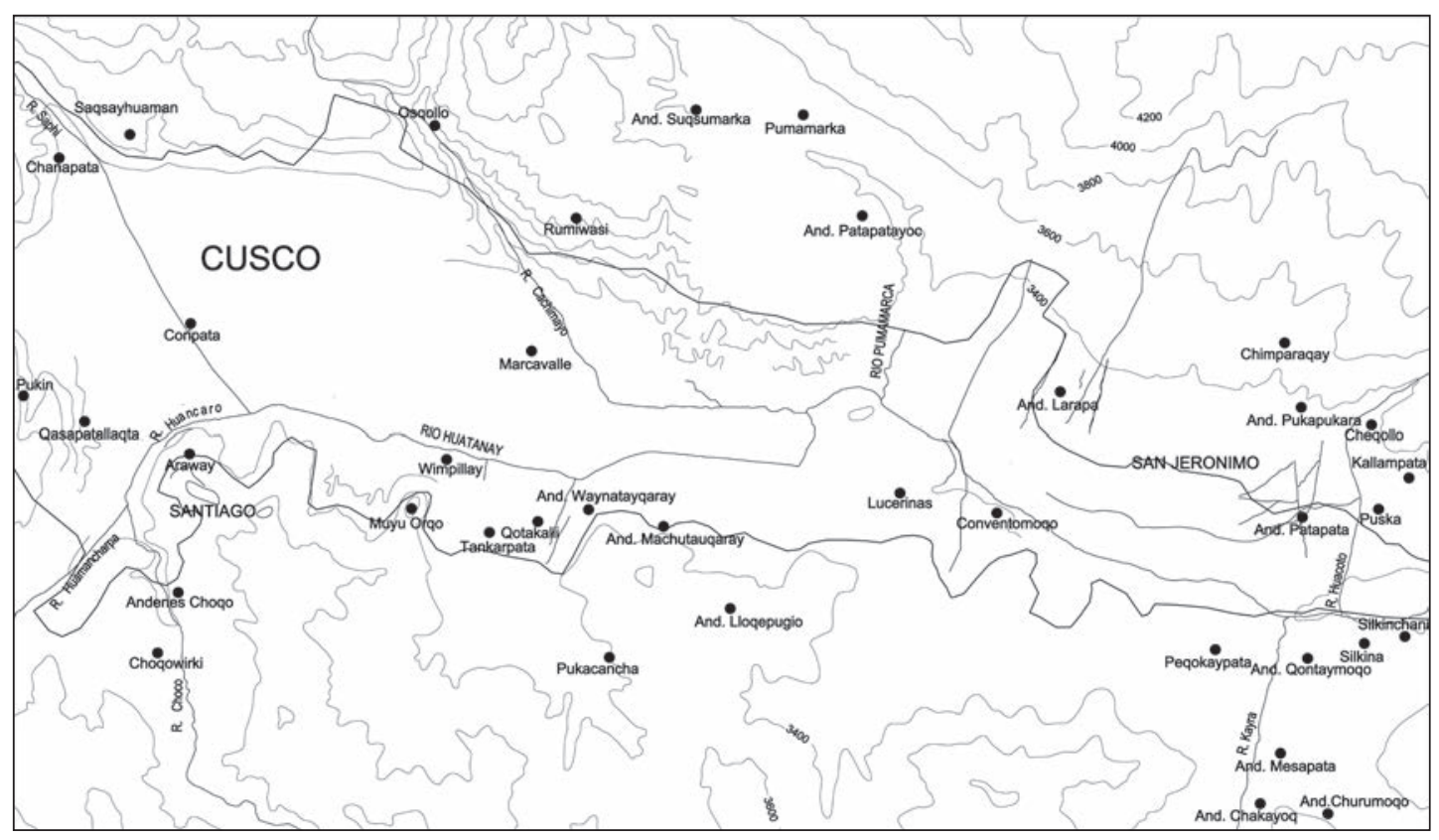

Figura 1. Sitios arqueológicos en el valle del Cusco

\section{Conventomogo}

El lugar se encuentra sobre una colina del cerro del mismo nombre a la margen derecha del río Huatanay, en el distrito de San Jerónimo, departamento y provincia del Cusco a 3290 msnm y coordenadas UTM: 186063 E y 8501157 N.

El sitio arqueológico comenzó a ser lotizado por la asociación de vivienda Altiva Canas desde el año 2002, con la apertura de calles niveladas con tractor y lotes construidos con casas de adobe en un $90 \%$ y con casas de concreto en un $10 \%$, actualmente solamente quedan algunos lotes vacios. Del sitio arqueológico han quedado algunas secciones de muros los cuales han sido recuperados mediante excavaciones arqueológicas en el lado este de la urbanización. El plano del Catastro Arqueológico del Ministerio de Cultura, Cusco, del año 2002 muestra algunas estructuras rectangulares y un conjunto de muros de aterrazamientos en el extremo sureste del cerro Conventomoqo. (Figs. 2 y 3 ). 


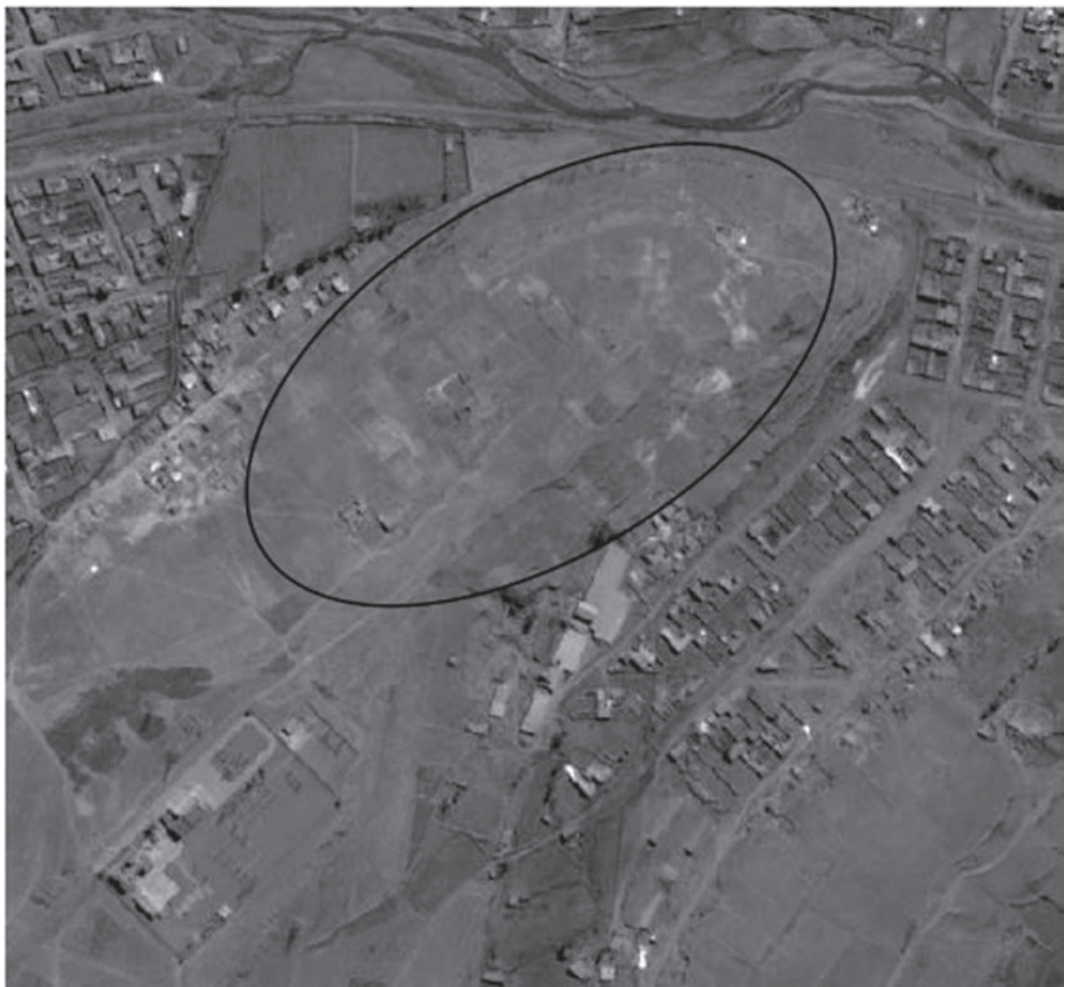

Figura 2. Vista del sitio de Conventomoqo en 2001 (cortesia de Google Earth).

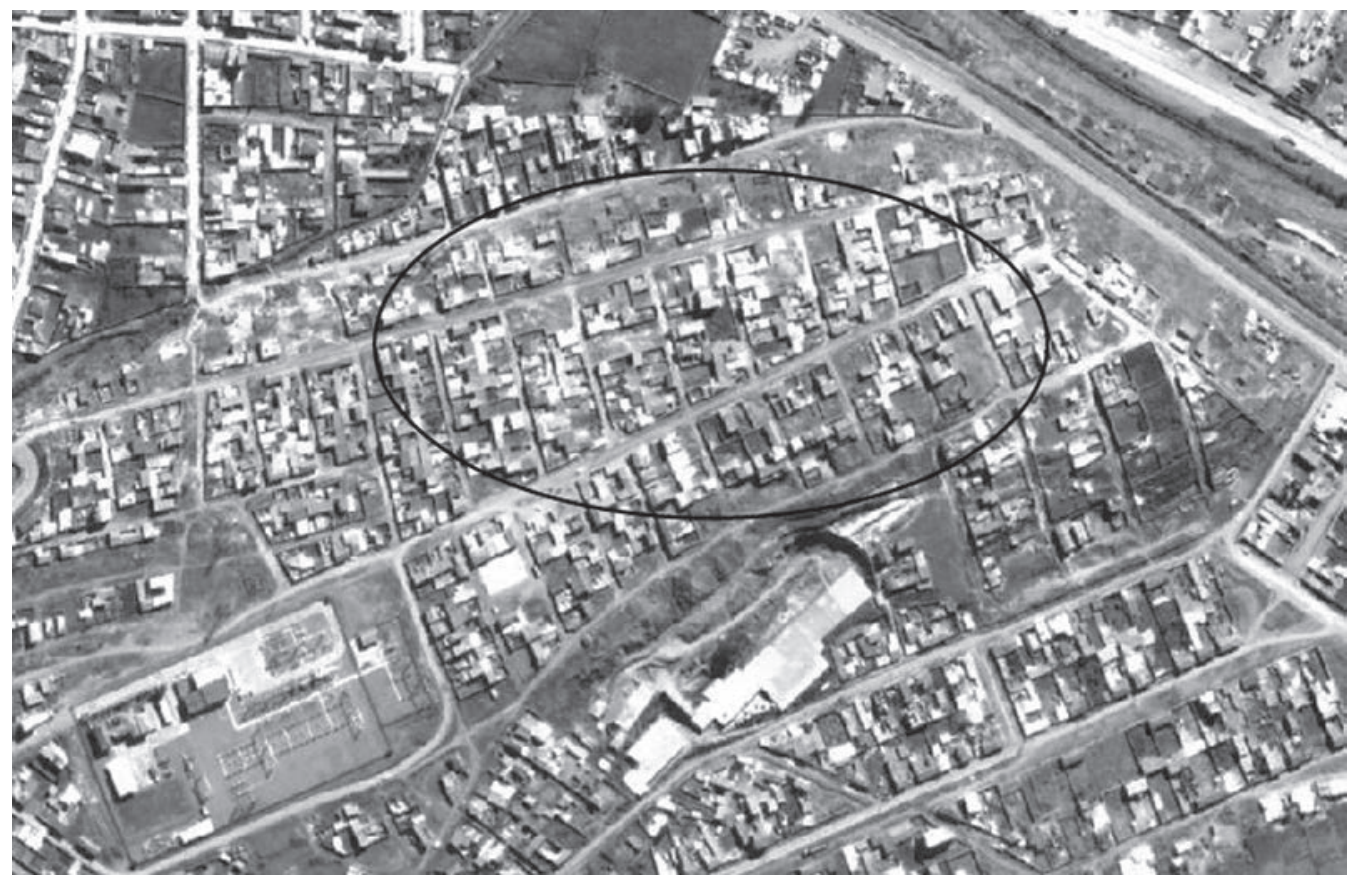

Figura 3. Vista del sitio de Conventomoqo en 2002 (cortesia de Google Earth). 


\section{LAS EXCAVACIONES ARQUEOLÓGICAS}

El año 2007 realizamos un trabajo de arqueología de salvataje en Conventomoqo y solo pudimos hacerlo en las zonas libres como parques, calles y algunos lotes de vivienda que todavía no habían sido construidos. Es por eso que, la información de las estructuras arquitectónicas identificadas es parcial, pero nos proporcionó información básica para plantear un proceso de ocupación del sitio.

Como parte del proceso de investigación, planteamos realizar excavaciones con 29 pozos de prueba (unidades de excavación) que nos permitió identificar algunas áreas con potencial arqueológico. El área total que abarcó la excavación fue de 348,40 m², demostrando que el lugar tuvo una ocupación continuada desde el periodo formativo Tardío (asociado a cerámica Chanapata derivado) hasta el periodo Inca-colonial.

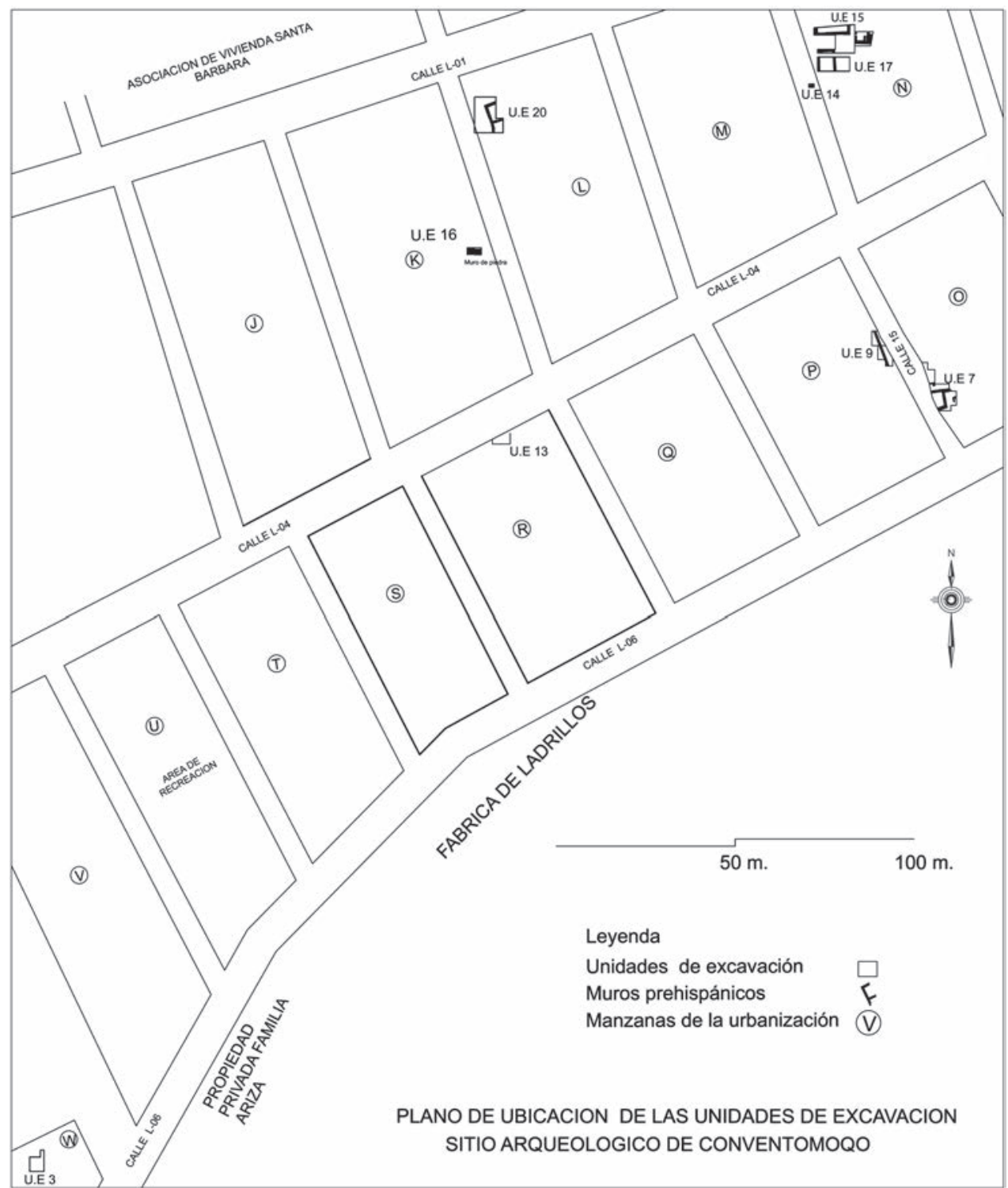

Figura 4. Ubicación de las unidades de excavación con las construcciones incas. 


\section{LA OCUPACIÓN INCA EN CONVENTOMOQO}

De acuerdo a las evidencias arqueológicas, el área que habrían ocupado las edificaciones inca debe haber abarcado una hectárea aproximadamente (100 m por lado); fuera de esta área el arqueólogo Raymundo Béjar Navarro en 1986 excavó en la actual Subestación Eléctrica de Electro Perú que se encuentra aproximadamente a $150 \mathrm{~m}$ al oeste de nuestra área de excavación, donde recuperó aproximadamente 150 entierros, llegando a la conclusión de que el lugar correspondería a un cementerio Inca (Farfán 2009: 133).

Por las características del asentamiento y de su arquitectura se puede establecer que el lugar fue una pequeña aldea con edificios dispersos asociados a algunas terrazas de cultivo. En base a las excavaciones realizadas se pudo identificar pequeñas secciones de muros que en algunos casos son de adobe en la parte superior, los que probablemente nos podrían dar algunos indicios de la planificación urbana y constructiva, sumado a estas evidencias tenemos el levantamiento topográfico que realizó el Instituto Nacional de Cultura - Cusco (actualmente Ministerio de Cultura) donde muestra un sistema de aterrazamientos hacia el extremo este de la colina y sobre la parte superior aparecen algunas estructuras rectangulares y cuadrangulares bastante grandes (Fig. 2). En el sitio arqueológico de Lucerinas, que se encuentra aproximadamente a un kilómetro al este (Farfán 2009), se pueden observar construcciones similares a las encontradas en Conventomoqo.

En base a la distribución de la arquitectura inca en la zona, se observa que estas estructuras no estuvieron de acuerdo a un sistema de canchas por oposición y simetría tal como lo define Bouchard (1976) más bien, los edificios fueron construidos sin ningún orden y asociados a algunos aterrazamientos que han desaparecido. Tipológicamente estas estructuras son rectangulares y cuadrangulares con muros de piedra simple, de aparejo irregular. Las difeferencias tipológicas y constructivas parece ser que se debieron a aspectos funcionales.

\section{EVENTOS DE ABANDONO DE CONVENTOMOQO}

En Conventomoqo se pudieron identificar evidencias de que este lugar fue abandonado parcialmente o en su totalidad a mediados del siglo XVI; el lugar empezó a ser destruido desde su abandono y continua hasta la actualidad. La información histórica es sumamente interesante para aproximarnos a la época donde se sucedieron todos estos eventos en las diferentes estructuras y unidades de excavación.

Cuando los españoles llegaron al Cusco en 1533 la organización politica inca se ve sumamente afectada. Durante 1539 a 1572 existía una tensión política debido a la resistencia de los últimos incas en Vilcabamba. En 1559 Polo de Ondegardo corregidor de la ciudad del Cusco recibe la orden del marqués de Cañete para que vea los sitios y ese mismo año organizó cinco parroquias de indios: la parroquia de San Sebastián, Carmenca ${ }^{1}$, Totocache ${ }^{2}$ Cayocache $^{3}$ y San Cristobal ${ }^{4}$ (Rowe 2003: 135-136). Es durante el gobierno del virrey Toledo, desde 1569 a 1581, que emprende la tarea de organizar el gobierno colonial, con una política de reasentamiento masivo de la población nativa (Rowe 2003: 247). En 1572 el virrey Toledo hizo una nueva reducción en ocho parroquia y juntaron a algunos ayllus y panacas que habrían estado en un mismo suyo como Sucsu panaca y Aucaille panaca que fueron reducidos a San Sebastián, pero a su vez mantenía las antiguas panacas de Chima y Raurau (Sherbondy 1986: 55).

1 Corresponde a la actual parroquia de Santa Ana, que se ubica al noroeste del Cusco.

2 Es la actual parroquia de San Blas.

3 Corresponde a la parroquia de Belén, pero su primigenia ubicación se habría situado en Coripata y que se mudo a su ubicación actual en el siglo XVII (Rowe 2003:138)

4 Se ubica en el actual barrio de San Cristóbal 
Es lógico pensar que el abandono de los asentamientos incas se dio durante este periodo de reasentamiento del gobierno del virrey Toledo, y la población de Conventomoqo habría pasado a ser parte de la parroquia de San Jerónimo, no tenemos el dato exacto de la toponimia actual, pero debemos indicar que el ayllu de Pillao Matao que es colindante a este lugar, es parte de la parroquia de san Jerónimo.

En la unidad de excavación 7, se encontraron varias secciones de muros que deben corresponder a varias estructuras arquitectónicas que no se lograron identificar tipológicamente; pero, adosadas a éstas se identificaron varios eventos que corresponden a abandonos, los cuales detallamos a continuacion:

Una estructura arquitectónica de forma zigzagueante que remata la parte superior con un muro de adobes (Fig. 6) fue rellenado uniformemente hasta una altura de 0,50 $\mathrm{m}$ donde se produce una incineración (Fig. 7); este nivel coincide con el inicio de arranque del muro de adobe. Esto nos abre

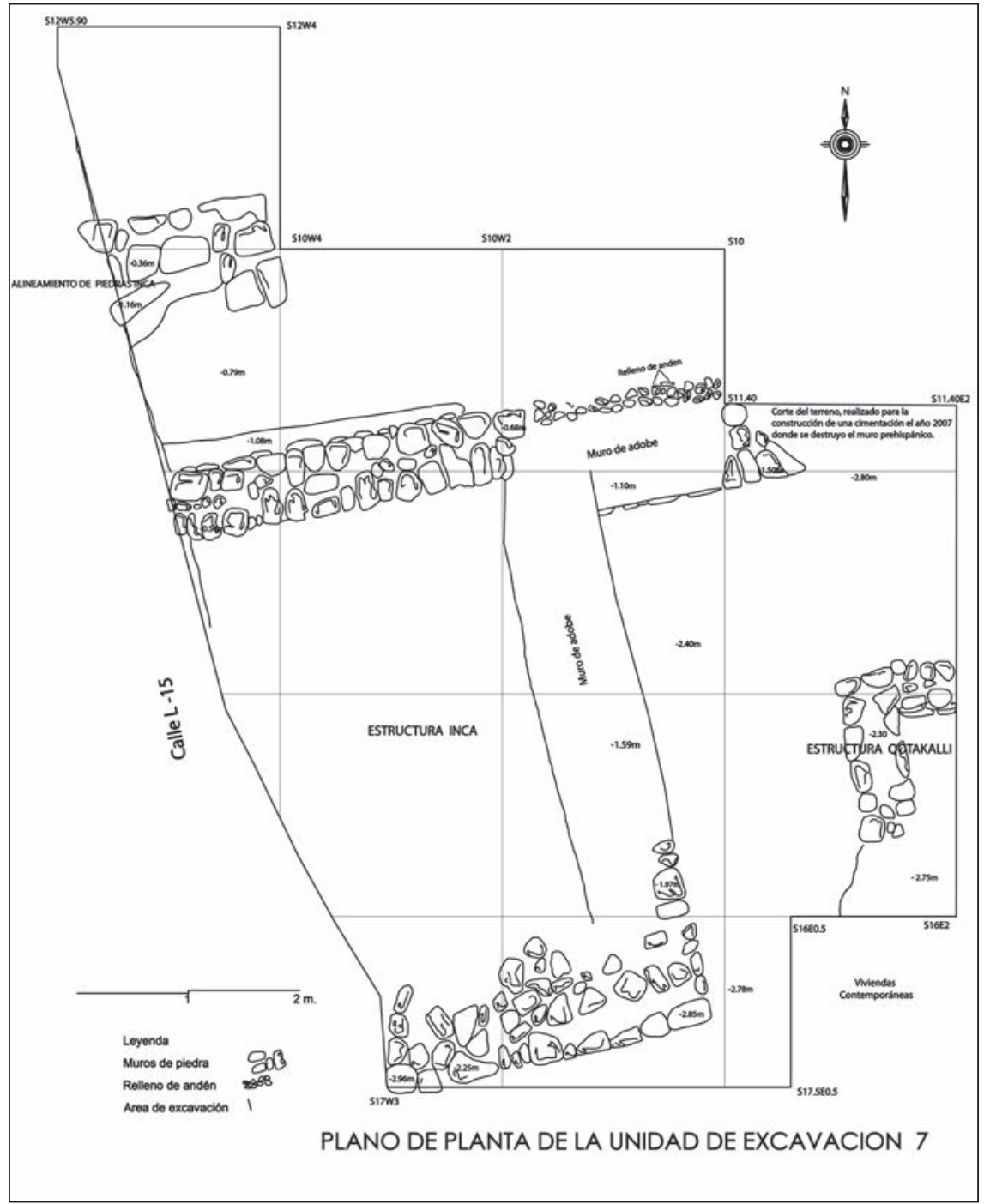

Figura 5. Detalle de las estructuras arquitectónicas de la unidad de excavación 7. 


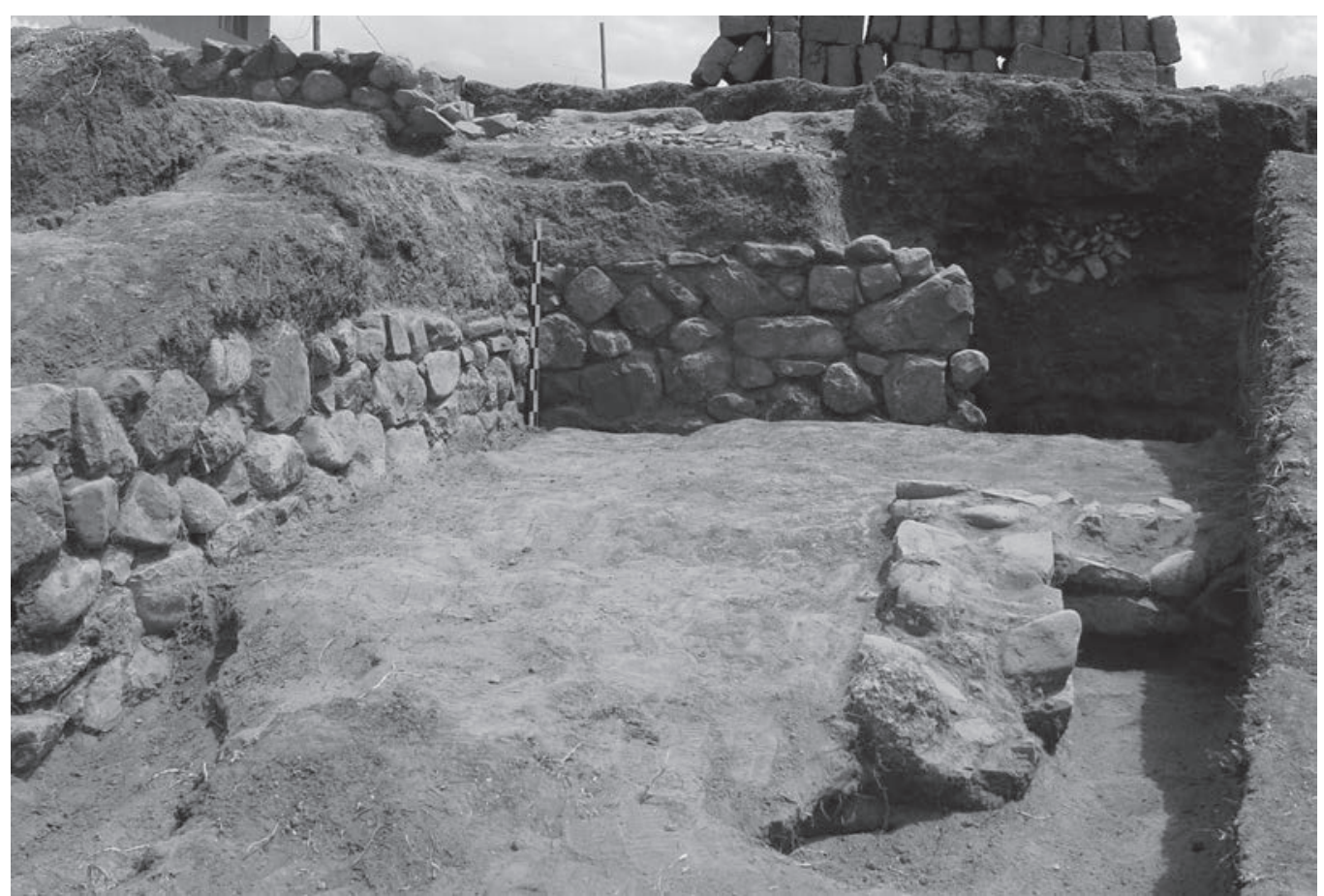

Figura 6. Vista del nivel de ocupación con un muro de piedra el cual remata con un muro de adobe.

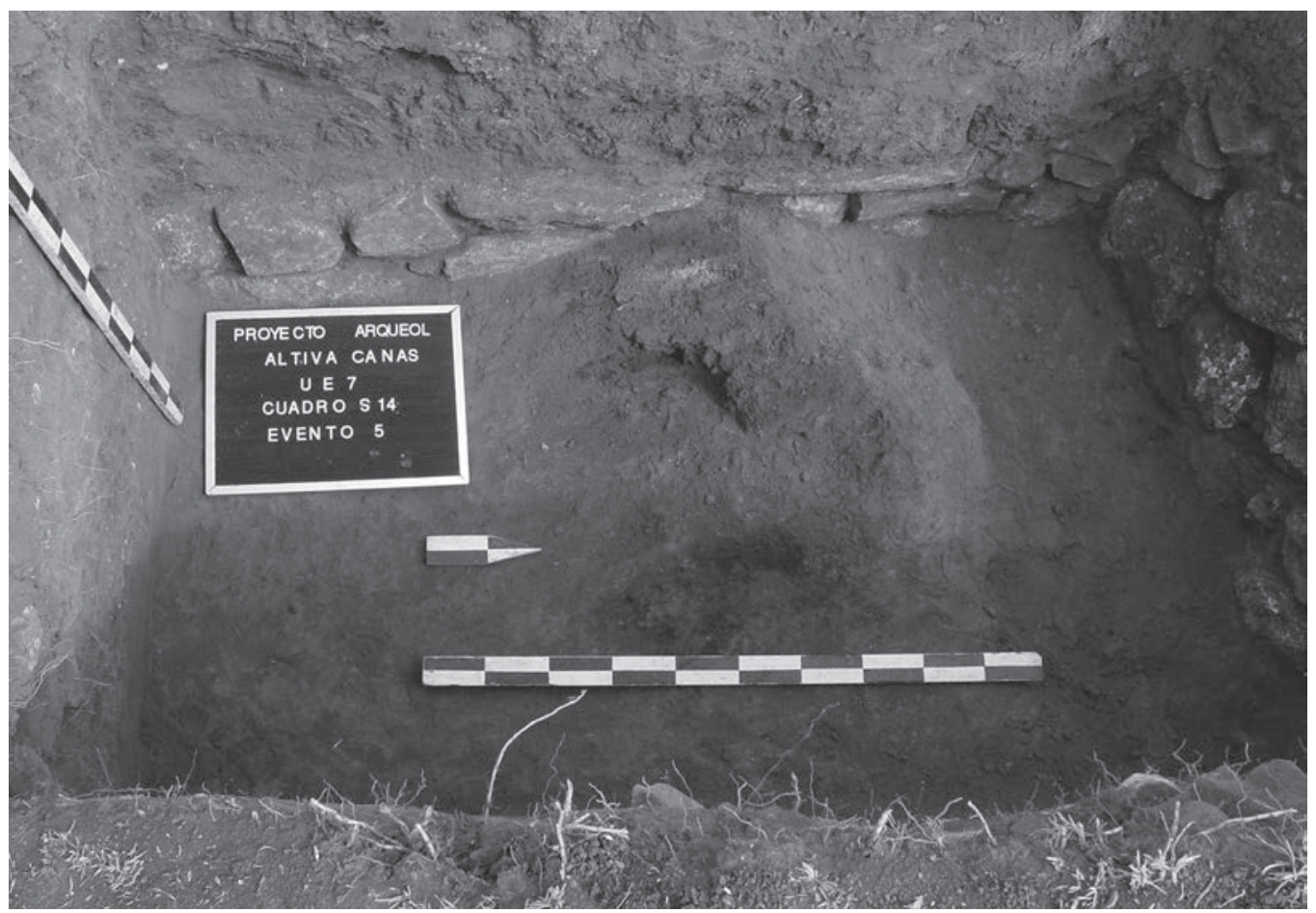

Figura 7. Incineración de material orgánico al nivel de la hilada superior del muro de piedra. 
varias posibilidades: la primera sería que es parte del techo de paja que cayó y fue incendiado, pero, solamente esta quema se encuentra en un área de 1 x $1 \mathrm{~m}$, si hubiese caído la techumbre, tendríamos evidencias del maderamem ${ }^{5}$ que habría sostenido el techo. La segunda posibilidad que este evento se llevó independientemente y el material orgánico fue quemado intencionalmente.

Por encima del nivel de incineración de la figura 7, adosada al muro norte de la estructura a la altura del muro de adobe en la cuadrícula S12 (por encima de todas las estructuras) tenemos la evidencia de un evento asociado a fragmentería de cerámica inca, huesos de camélidos y piedras sueltas de los muros. La cerámica inca decorada corresponde al 67,4\% y la cerámica doméstica al 32,6\%, correspondiendo generalmente a cuerpos de aríbalos, cuencos y ollas en poca proporción (Fig. 8).

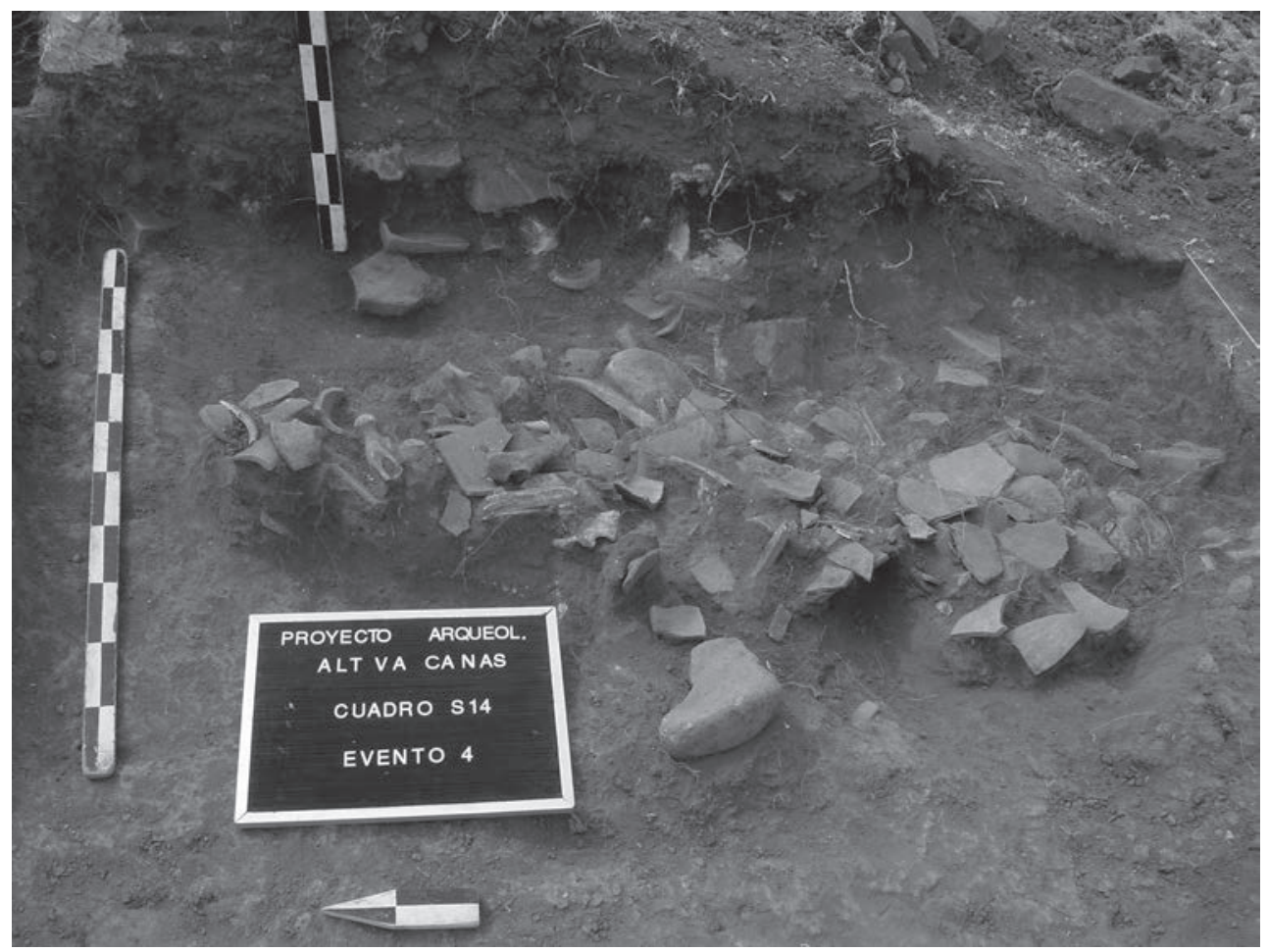

Figura 8. Evento de un probable "festín".

El cubrir o enterrar una infraestructura lleva a varias acciones como lo hemos detallado antes, podríamos estar solamente ante un evento para cubrir las estructuras y abandonar este espacio, o estos sucesos también se daban con algún tipo de ritualidad. No tenemos la evidencia absoluta para afirmar que es parte de un festín. Varios trabajos llevados a cabo en el área andina analizan a la fiesta en base a sus residuos de comida y de bebida asociados a arquitectura pública (Véase Ikehara y Shibata 2005; Makowski et al. 2005; McEwan et al. 2005, Vega-Centeno 2005 y Delgado 2013).

El acto de romper los objetos de uso diario o ceremonial está documentado en el área andina desde la época preinca, Mc Ewan et al. (2005: 273) en sus excavaciones en la huaca 1 y 2 de Choquepukio sugiere el rompimiento ritual de algunas vasijas para cubrir la huaca, en el Cerro Blanco de Cahuachi se brindaban ofrendas de cerámica polícroma que fueron rotas intencionalmente (Llanos 2010:41), en

5 Maderamem: Conjunto de maderas que sirven para un edificio o que entran en una construcción (Enciclopedia Universal Salvat 2009: 9429) 
la estructura 4 de Cerro Baúl el evento ritual consistió en quebrar vasijas de cerámica sobre el piso de la galería (Williams e Isla 2002: 93) y para la época inca tenemos algunos reportes en Urqo, a las afueras de la ciudad de Calca, (Rosa 2008: 78-79) supone que el rompimiento de cerámica corresponde el punto final de la ocupación. El acto de romper las vasijas podría entenderse como un acto de sacrificio, el punto final del ritual y la renovación (Kaulicke 2005: 399). Como podemos observar, el abandono y el cubrir este edificio no se dio con un solo acto, por lo menos hemos podido identificar tres acciones, lo que no sabemos es si estos actos fueron realizados con una diferencia corta de tiempo o fueron realizados paulatinamente.

Otro evento que nos indicaría que el lugar fue abandonado lo tenemos a tres metros del evento anterior en el cuadro S10W4 y consiste en una acumulación de piedras sueltas descontextualizadas asociadas a pedazos de ollas de cerámica Inca y a una fuente volteada de piedra de pórfido granodioritico (Fig. 9), la fuente presenta sus bordes fracturados ex profesamente y no por uso o desgaste durante su vida útil, sino que fue desmembrada para inutilizarla; el evento fue colocado y posteriormente fue rellenado con tierra de las proximidades, por eso se tiene cerámica inca y formativa mezclada.

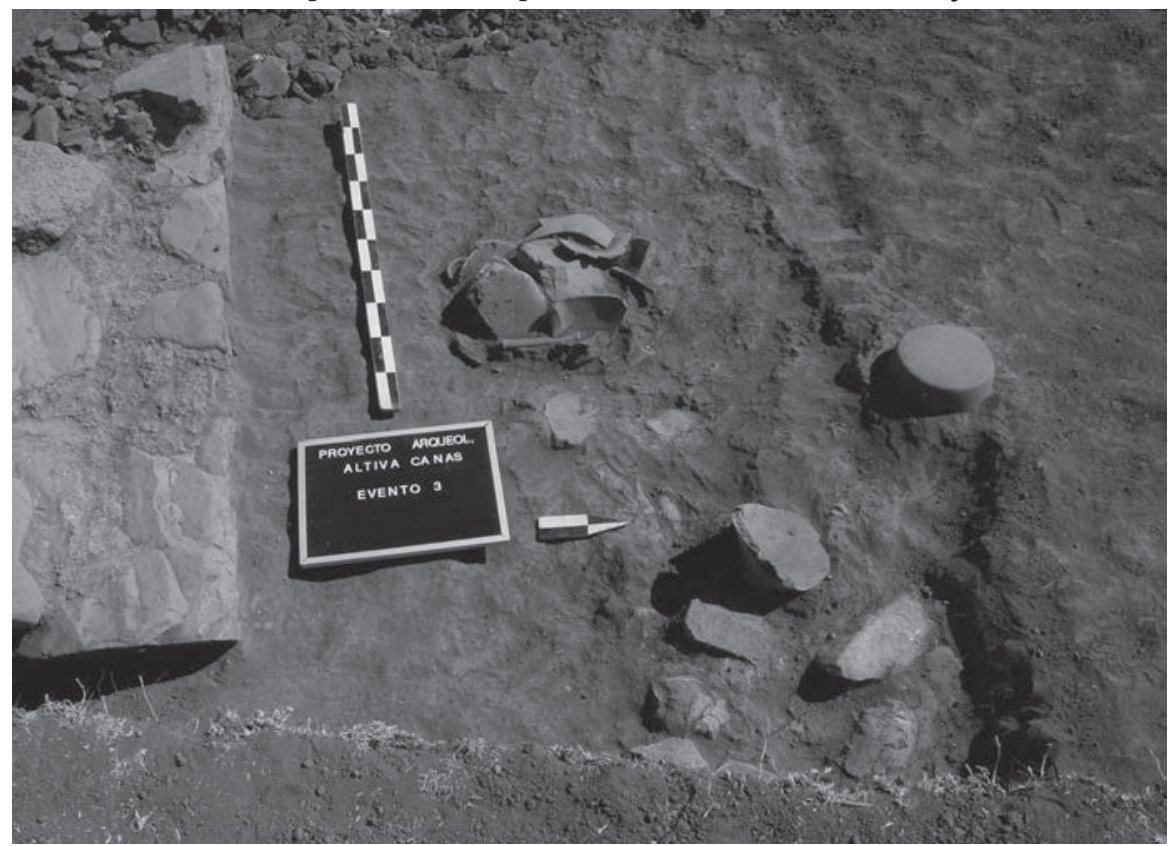

Figura 9. Nótese la acumulación de piedras, fragmentos de cerámica Inca y una fuente de piedra volteada.

Las evidencias arqueológicas en el periodo Inca con relación a enterrar objetos volteados lo tenemos en el sector de Pukara en Tipón (Delgado 1998) y en Arcopata (Delgado 2013: Fig. 13).

En la unidad de excavación 9 se pudo recuperar el lado oeste de una estructura rectangular de $10.50 \mathrm{~m}$ de largo, esta estructura por sus características morfológicas podría corresponder a una Kallanca (Fig. 10). Esta estructura fue rellenada aproximadamente $1 \mathrm{~m}$ desde su piso original con tierra mezclada con fragmenteria de cerámica inca, formativa y algunos pedazos de bloques de tierra quemada probablemente del mortero de las paredes interiores.

Las Unidades de excavación № 15 y 17 se encuentran hacia el norte en la ladera del cerro que mira hacia la ciudad del Cusco (Fig. 11). Estas tres estructuras rectangulares se construyeron sobre la ocupación formativa y forman un espacio interior que dan la apariencia de una kancha y se encuentran distribuidas ortogonalmente. La estructura rectangular que se encuentra hacia el este es de 3,40 x 4,50 m, la segunda que se encuentra hacia el norte es de 9,65 x $3 \mathrm{~m}$, es muy probable que fuese un 


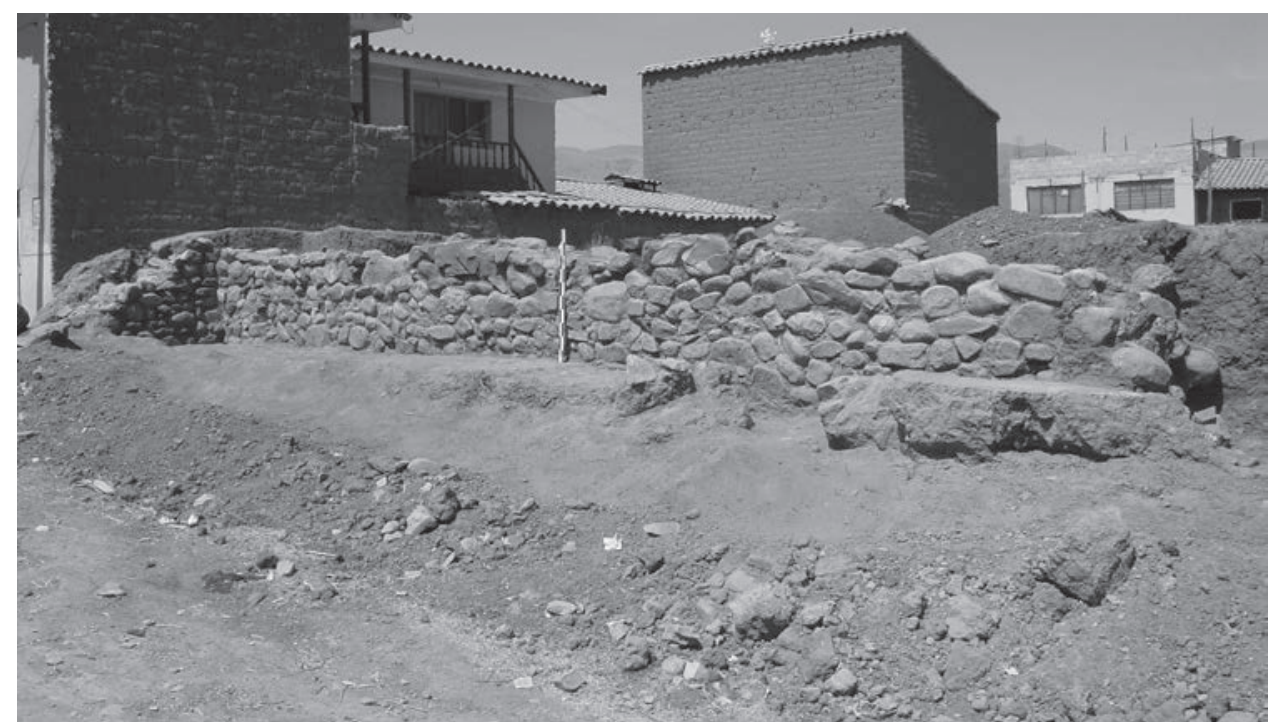

Figura 10. Estructura rectangular recuperada la cual fue tapada con tierra.

poco más grande, con un piso de Qontay, esta estructura se encuentra a nivel de cimentación (Fig. 12). La que se encuentra hacia el sur es de $6,25 \times 5 \mathrm{~m}$, su lado norte-sur debe ser mucho más grande ya que se encuentra cortada por la construcción de una vivienda contemporánea. Estas edificaciones no presentan evidencias de haber sido cubiertas intencionalmente.

El fogón 3 (Fig. 11) nos proporcionó un fechado radiocarbónico bastante tardío, lo cual es interesante ya que nos ofrece una data que correspondería al periodo Inca-Colonial temprano y coincide con la idea que las poblaciones siguieron ocupando este lugar hasta la reducción de Toledo años después.

$\begin{array}{lcccc}\text { Muestra } & \text { Material } & \text { Años C14 } & \text { AD/BC } & \\ \text { AA101204 } & \text { Madera } & 404 \pm 32 & 95 \% & 1452-1627 \mathrm{cal} \mathrm{AD}^{6}\end{array}$

El material botánico analizado de este fogón nos brindó la información de los productos que se seguían consumiendo como quinua y kiwicha?.

La última estructura rectangular doméstica es la que se encontró en la unidad de excavación 20 , construida con cantos rodados de 5,60 x 3,50 m, la cual fue modificada agregándole una sección al muro oeste. Adicionalmente el muro sur fue modificado durante este periodo reduciéndolo de 0,65 a $0,55 \mathrm{~m}$ de ancho. Como en los casos anteriores estas estructuras se construyeron sobre una ocupación formativa. El muro norte se desplomó al exterior hacia la ladera del cerro, un primer estrato al interior de la estructura rectangular es una capa de 0,40 m de tierra semicompacta con cerámica inca y formativa, no presenta eventos como fogones, áreas de combustión u otros elementos domésticos o rituales. Un segundo nivel que se da a la altura de la cabecera del muro consistió en cubrirlo con tierra y los mampuestos de los muros formando un aparente caos (Fig. 13).

La intrusión 1, que corresponde a un hueco en el suelo geológico de 2,6 x 1,7 m con una profundidad de 0,25 m pegada al muro norte de la estructura (Fig. 13), la cual estuvo rellenada con pedazos

6 Laboratory number: AA101204 NSF Arizona AMS Laboratory

7 Rojas Velásquez 2011:Muestra 02 


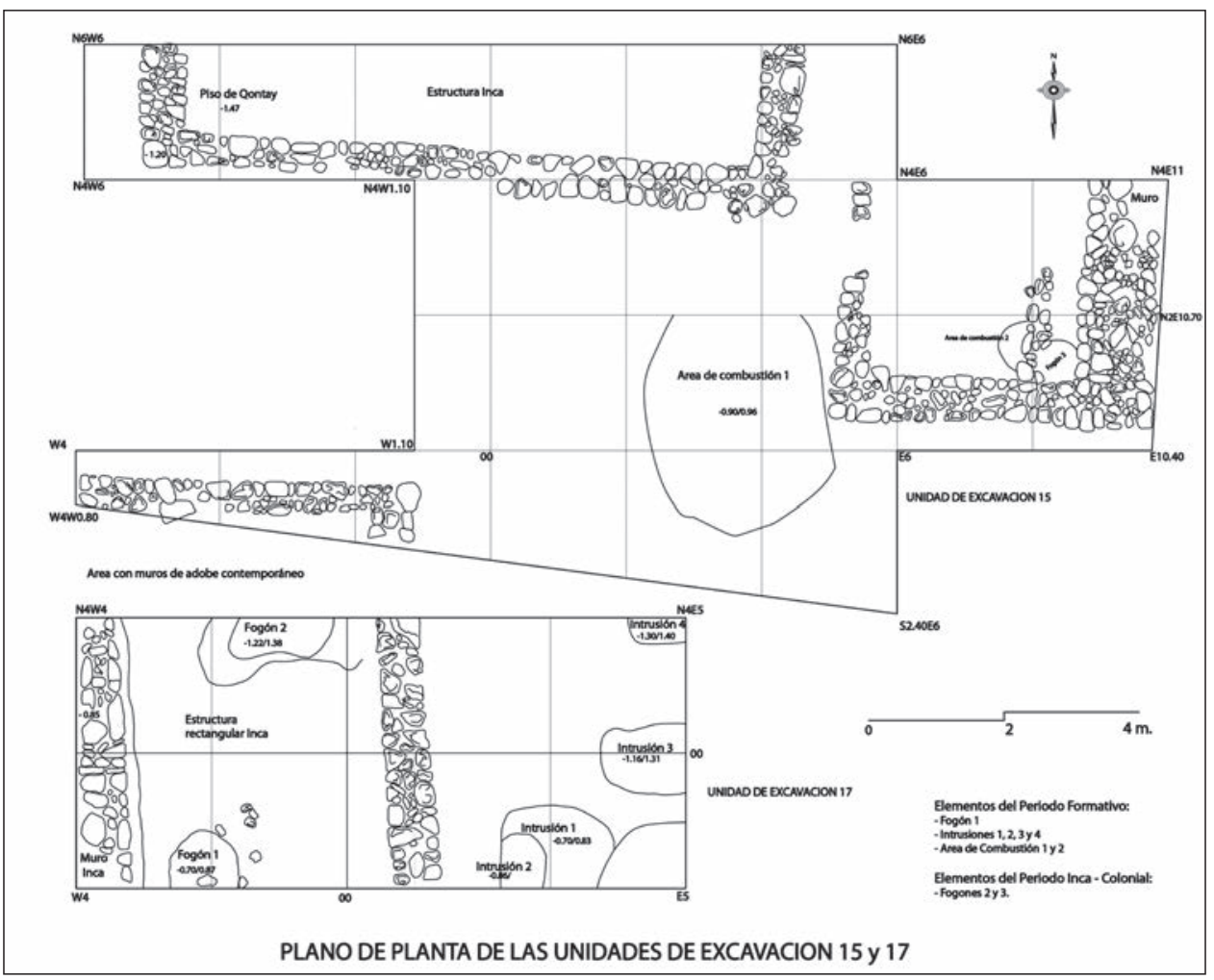

Figura 11. Plano de planta de las Unidades de excavación 15 y 17.

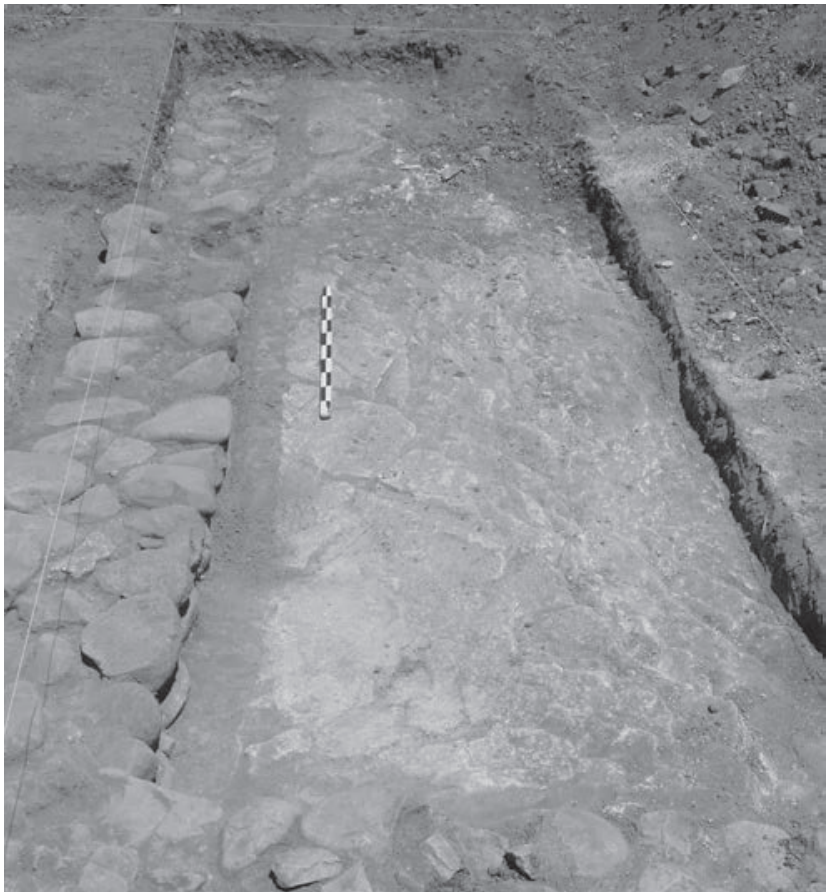

Figura 12. Detalle de la cimentación de la estructura rectangular Inca. 


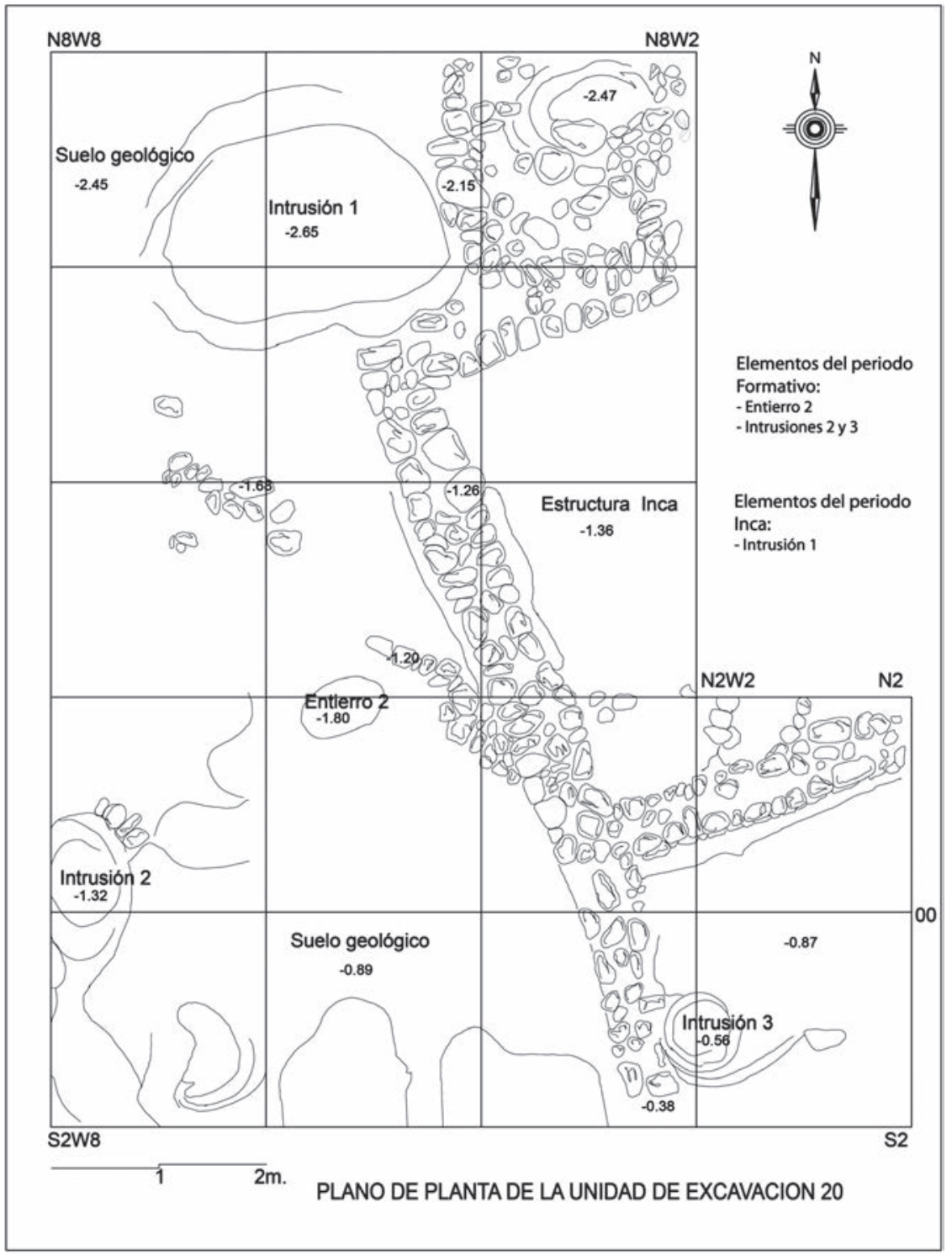

Figura 13. Plano de planta de la unidad de excavación 20.

de vasijas Incas dispuestos en forma desordenada y mezclada con bloques líticos de los muros del edificio; estos fragmentos no corresponden a uno o varios objetos que fueron rotos in situ, más bien corresponden a pedazos independientes de ceramios colocados intencionalmente. La cerámica decorada de este evento representa el $71 \%$ y la cerámica no decorada el 29\%; del total de la fragmentería el $83 \%$ corresponde a cuerpos de vasijas grandes y el resto a aríbalos, platos y vasos.

Adosado al muro sur se ubicó un área de combustión y el análisis botánico determinó restos de maíz (Zea mays) de tipo amiláceo, semillas carbonizadas de chenopodium quinoa, fragmentos de frutos carbonizados de Erythoxylum coca L., Magnoliópsida y Liliópsida (que podría corresponder a maíz y cañas) ${ }^{8}$.

8 Bertoni 2012. 


\title{
Discusión
}

Conventomoqo una aldea inca en el valle del Cusco, no es uno de los sitios más grandes, mas bien corresponde a una pequeña población que se asentó en la margen derecha del río Huatanay, la cual nos ha proporcionado información interesante para entender parte de la vida cotidiana, los productos alimenticios que consumían como maíz, papa, quinua, kiwicha y coca que se han identificado, éstos a su vez no son excluyentes de otros productos que también debieron haber consumido.

Todos estos edificios inca presentan algunas cosas en común que corresponden a áreas domésticas y que presentan diferentes eventos que se han llevado en ellas como parte del abandono del lugar y es probable que estos acontecimientos se hayan dado cuando los españoles se asentaron en el Cusco Incaico o posteriormente cuando se dan las reducciones.

Los pueblos en el valle del Cusco inicialmente fueron reducidos a cuatro pueblos o parroquias Carmengo, Cocampata, Cavicache y Totocache (Málaga 1975: 26) y que posteriormente una nueva reducción en 1572 creó ocho parroquias. Es importante señalar que fue política del nuevo gobierno español reubicar a las poblaciones de acuerdo a las parroquias, es lógico pensar que las poblaciones que vivieron en Conventomoqo fueron reducidos a una de estas parroquias, el fechado radiocarbónico que se obtuvo no nos indica cuando fue abandonado, pero, si nos indica que a mediados del siglo XVI el lugar seguía siendo ocupado.

Las evidencias de los eventos de abandono, que fueron descritos, no nos dicen exactamente cuando se realizaron, pero, la cerámica inca sigue siendo idéntica a la cerámica producida antes de la llegada de los españoles, no presenta evidencias de torno, ni modificaciones o intrusión de nuevos elementos decorativos. Tampoco en los estratos arqueológicos tenemos pruebas de arquitectura, cerámica u otros productos que tuvieran algún indicio de época colonial.

\section{BiBLIOGRAFIA}

\author{
ARDILES, Percy \\ 1986 «Sistema de drenaje subterráneo prehispánico». Allpanchis 27: 75-97 año XVIII, Cusco. \\ BARREDA, Luis \\ 1973 Las culturas Inka y Pre Inka del Cusco. Tesis para optar el grado de Doctor en Letras y Ciencias Huma- \\ nas. Universidad Nacional San Antonio Abad del Cuzco. \\ BAUER, Brian \\ 2008 Cuzco Antiguo: Tierra natal de los Incas. Cusco: Centro Bartolomé de las Casas. \\ BENAVIDES, María y María VIZCARRA \\ 2010 Arqueología de Raqay Raqayniyoc, San Jeronimo - Cusco. Tesis para optar al grado de licenciado en \\ arqueología. Universidad Nacional de San Antonio Abad del Cusco. \\ BERTONI, Gabriela \\ 2012 ms Informe Arqueo botánico Altiva Canas, Cusco. Museo de Historia Natural UNMSM - LIAP Labo- \\ ratorio de Investigaciones Arqueo botánicas del Perú. \\ BOUCHARD, Jean-Francois \\ 1976 «Patrones de agrupamiento arquitectónico del Horizonte Tardío del valle del Urubamba». Revista \\ del Museo Nacional, XLII: 97-111, Lima. \\ CANDÍA, Alfredo \\ 1992 Arquitectura de Qhataqasapatallaqta. Tesis para optar al Grado de Licenciado en Arqueología. Uni- \\ versidad Nacional de San Antonio Abad del Cusco. \\ 1995 «Ocupación killke en Qhata Q'asallaqta. Saqsaywaman». Revista Arqueológica 4: 122-132. Instituto \\ Nacional de Cultura Departamental Cusco.


CLAROS, Dorina y Alfredo MORMONTOY

1992 Arqueología de Qontaymoqo y Silkinchani (San Jerónimo - Cusco). Tesis para optar al Grado de Licenciado en Arqueología. Universidad Nacional de San Antonio Abad del Cusco.

CUMPA, Claudio

2001 Informe de Investigación Arqueológica Silkinchani. Instituto Nacional de Cultura Cusco.

DEL PEZO, Marco

2002 Informe de Investigación Arqueológica Qotakalli. Instituto Nacional de Cultura Cusco.

DELGADO, Carlos

1998 «Excavaciones arqueológicas en Tipón (Pukara) Parafernalia de una ofrenda Inca». Revista Saqsaywaman 5: 147-163. Cusco: Instituto Nacional de Cultura.

2013 «Feasts and offerings in Arcopata, Cusco». Andean Past 11.

ENCICLOPEDIA UNIVERSAL SALVAT

2009 Enciclopedia universal. Volumen № 20, Madrid: Editorial Salvat.

FARFÁN, Domingo

2009 «Investigaciones arqueológicas en Lucerinas». Saqsaywaman 9: 127-148. Cusco: Instituto Nacional de Cultura.

GUILLÉN, Jorge

2009 «Investigación Arqueológica en Salonniyoq Templo de la Luna». Saqsaywaman 9: 52-71. Cusco: Instituto Nacional de Cultura.

IKEHARA, Hugo y Koichiro SHIBATA

2005 «Festines e integración social en el periodo formativo: Nuevas evidencias de cerro Blanco, valle bajo de Nepeña». Boletín de Arqueología PUCP 9: 123-160. Lima.

KAULICKE, Peter

2005 «Las fiestas y sus residuos: Algunas reflexiones finales». Boletín de Arqueología PUCP 9: 387-402, Lima.

LLANOS, Luis A.

1936 «Trabajos Arqueológicos en el departamento del Cuzco». Revista del Museo Nacional 5(2): 123-156. Lima.

LLANOS, Oscar

2010 «Cahuachi: residencia y paisaje sacralizado de un centro político nazca». Revista Española de Antropología Americana, (40)1: 27-51.

MAKOWSKI, K., CÓRDOVA, M., HABETLER, P., LIZÁRRAGA M.

2005 «La plaza y la fiesta: reflexiones acerca de la función de los patios en la arquitectura pública prehispánica de los periodos tardíos». Boletín de Arqueología PUCP 9: 297-334, Lima.

MÁLAGA, Alejandro

1975 «Las reducciones en el Virreinato del Perú 1532-1580». Revista de Historia Americana 80: 9-42. Instituto Panamericano de Geografía e Historia.

MC EWAN, G., GIBAJA, A., CHATFIELD, M.

2005 «Arquitectura monumental en el Cuzco del Periodo Intermedio Tardío: evidencias de continuidades en la reciprocidad ritual y el manejo administrativo entre los horizontes Medio y Tardío». Boletín de Arqueología PUCP 9: 257-280, Lima.

NILES, Susan

1984 «Architectural form and social function in Inca towns near Cuzco». En Ann Kendall, ed. Current archaeological projects in the Central Andes: Some approaches and results. 205-223- BAR International Series 210, Oxford.

PARDO, Luis A.

1939a «Hacia una nueva clasificación de la cerámica cuzqueña del antiguo imperio de los Incas, Perú». Revista del Instituto Arqueológico del Cusco. 3(4-5): 1-22. Cusco.

1939b «Arte Peruano: Clasificación de la cerámica cuzqueña (época incaica)». Revista del Instituto Arqueológico del Cuzco 4(6-7): 3-26, Cusco. 
1957 Historia y Arqueología del Cuzco. Vol. 2. Imprenta del Colegio Militar Leoncio Prado, Lima.

PILARES, José

2002 Informe de Investigación Arqueológica Qhataqasapatallaqta. Instituto Nacional de Cultura Cusco.

PILCO, René

2005 Informe anual de Investigación de Emergencia zona Arqueológica de Muyu Orqo. Instituto Nacional de Cultura Cusco.

QUISPE, Rita

2009 Informe final Proyecto de emergencia Arqueológica obra: Pavimentación vías de acceso AAHH Arahuay, 1ro de enero. Proyecto Especial Plan Copesco.

ROJAS, Eliana

2011 ms Informe con resultados de análisis de rastreo e identificación de materia orgánica en muestras del PEA Altiva Canas - Cusco. Informe № 003-2011-ERV-DFQ-SDI-DIC-DRC-C/MC

ROSA, Maritza

2008 «La ocupación Inka en Urqo - Calca: una visión de su función y abandono a través de un contexto ritual». Saqsaywaman 8: 72-84, Instituto Nacional de Cultura Dirección Regional de Cultura Cusco.

ROWE, John H.

1944 An introduction to the archaeology of Cuzco. En: Papers of the Peabody Museum of American Archaeology and Ethnology, 10-23. Harvard University, Cambridge, MA.

2003 «El Barrio de Cayau Cachi y la parroquía de Belén». En: Los Incas del Cuzco siglos XVI-XVII-XVIII. 135142. Instituto Nacional de Cultura, Región Cusco.

SHERBONDY, Jeanette

1986 «Los ceques: códigos de canales en el Cusco incaico». Allpanchis 27: 39-74, año XVIII, Cusco.

UGENT, Donald y Carlos OCHOA

2006 La Etnobotánica del Perú, desde la prehistoria al presente. Concytec, Lima Perú

VALCÁRCEL, Luis E.

1934 «Los trabajos arqueológicos del Cusco. Sajsawaman Redescubierto II». Revista del Museo Nacional Tomo III: 3-36, 211-233. Lima.

1935 «Los trabajos arqueológicos en el departamento del Cusco. Sajsawaman Redescubierto III y IV». Revista del Museo Nacional Tomo IV: 1-24, 161-203. Lima.

VEGA-CENTENO, Rafael

2005 «Consumo y ritual en la construcción de espacios públicos para el periodo Arcaico Tardío: el caso del cerro Lampay». Boletín de Arqueología PUCP 9: 91-122. Lima.

WILLIAMS, Patrick y Jhony ISLA

2002 «Investigaciones arqueológicas en Cerro Baúl, un enclave Wari en el valle de Moquegua». Gaceta Arqueológica Andina 26: 87-120 INDEA, Lima. 